\title{
Pneumococcal capsular serotypes and lung infection
}

\section{Jeremy Stuart Brown}

Streptococcus pneumoniae is second only to Mycobacterium tuberculosis as a bacterial cause of worldwide mortality. Unlike $M$ tuberculosis, $S$ pneumoniae remains a major cause of death in the developed world, with a standardised mortality rate of 25 per 100000 in the UK. S pneumoniae is the commonest pathogen causing community acquired pneumonia (CAP), ${ }^{1}$ and the majority of serious $S$ pneumoniae infections are cases of CAP in infants and older people. $S$ pneumoniae is also an important cause of septicaemia, meningitis and infective exacerbations of chronic obstructive pulmonary disease and bronchiectasis. $S$ pneumoniae is surrounded by an extracellular layer of polysaccharide called the capsule which promotes immune evasion and is an essential virulence factor. ${ }^{2}$ The structure of the capsule differs between $S$ pneumoniae strains, with 93 variants identifiable by serotyping. Serotype prevalence is unequal, with the majority of disease caused by around 20 common serotypes. Which serotypes are predominant varies with age, geography and site of infection (eg, the nasopharynx, pleural space or the blood). In the paper by Bewick et al, technical advances in microbiology have been used to identify which $S$ pneumoniae capsular serotypes are the commonest causes of CAP in a UK centre. ${ }^{3}$ Why do these rather technical data matter to a clinician, and how could they affect future developments in the management of $S$ pneumoniae lung infection?

First, the ability to identify which serotypes are causing CAP is in itself a major advance. Previously, capsular serotyping required cultured bacteria and was therefore biased towards serotypes that cause septicaemia as respiratory cultures have such low sensitivity in CAP. For example, in Bewick et al's paper only 58 of 366 cases of $S$ pneumoniae CAP had positive cultures, mainly from blood. ${ }^{3}$ By using urine samples for a highly sensitive multiplex immunoassay based on xMAP bead technology, Bewick et al were able to identify $S$ pneumoniae capsular serotype in

Correspondence to Centre for Respiratory Research Department of Medicine, University College Medical School, Rayne Institute, London WC1E 6JJ, UK; jeremy.brown@ucl.ac.uk culture negative cases of CAP, providing for the first time a largely unbiased assessment of which serotypes cause CAP. At present the multiplex serotype immunoassay is restricted to only 14 serotypes, the pneumococcal conjugate vaccine (PCV13) serotypes and serotype 8, and this limitation is probably why no serotype was identified for one-third of their patients with $S$ pneumoniae CAP. Future technical developments are likely to increase the numbers of serotypes that can be detected and partially overcome this problem. Interestingly, the multiplex immunoassay increased the sensitivity of microbiological confirmation of $S$ pneumoniae CAP, identifying 144 cases in which the established urinary antigen test (Binax NOW, Portland, Maine, USA) was negative. ${ }^{3}$ This provides a potential justification for using this technology independent of the possible advantages in identifying serotype.

Second, capsular serotype could identify patients at risk of specific complications of CAP. Serotype affects $S$ pneumoniae resistance to complement, phagocytosis, adhesion to epithelial cells and virulence in mouse models. ${ }^{4-6}$ Probably related to these biological effects of serotype, in human disease duration of $S$ pneumoniae colonisation, the number of episodes of invasive disease per colonisation event, mortality and ability to cause empyema are all linked to serotype. ${ }^{7-9}$ For example, before the introduction of infant vaccination the top five capsular serotypes causing $S$ pneumoniae septicaemia and meningitis were 14, 19F, 6B, 23F and 18C, yet $80-90 \%$ of cases of $S$ pneumoniae empyema are caused by serotypes $1,3,7 \mathrm{~F}$ and 19A. ${ }^{9}$ Until now it was not clear whether the serotypes commonly causing empyema simply reflected their dominance as serotypes causing CAP or a specific affinity for invading the pleural space. Bewick et al found that only one of the three commonest CAP serotypes frequently causes empyema, and overall only $40 \%$ of cases of CAP were caused by serotypes associated with empyema. Similarly a recent Spanish study which used a multiplex PCR to identify $S$ pneumoniae serotypes in culture-positive patients with CAP showed serotypes 4, 5 and 8 are more likely and serotypes $19 \mathrm{~F}$ and $6 \mathrm{~B}$ less likely to cause an associated septicaemia. ${ }^{10}$ In addition, infection with serotype 3 is clearly associated with increased mortality. ${ }^{8}$ These data demonstrate specific $S$ pneumoniae serotypes are associated with important complications of CAP such as empyema, septicaemia and death. Identifying infecting serotype could therefore help risk stratify patients with $S$ pneumoniae CAP, and identify those who might benefit from earlier intervention such as drainage of pleural effusions. Furthermore, comparing the biology of serotypes associated with specific disease phenotypes will help characterise the bacterial and host factors involved in the pathogenesis of infection at different sites.

Third, Bewick et al have provided additional data suggesting that $S$ pneumoniae infection in younger patients is largely restricted to a limited number of highly invasive serotypes. As discussed above, serotype affects the ability of $S$ pneumoniae to cause invasive disease with attack rates (number of cases of meningitis or septicaemia per colonisation episode) ranging from 0 to 75 for different serotypes. Bewick et al found a direct correlation between increasing age and comorbidities and infection with less invasive serotypes, supporting the hypothesis that advancing age allows less aggressive $S$ pneumoniae serotypes to cause disease. ${ }^{3}$ The increasing incidence of less invasive serotypes with age and comorbidity (both markers for increased mortality) may also explain the seemingly paradoxical observation that infection with more invasive serotypes has a lower mortality than infection with less invasive serotypes. One caveat to these data is that the exclusion of serotype 14 and 19A strains from the invasive serotype group by Bewick et al could be challenged as both serotypes have high attack rates $^{7}$; including these serotypes in the invasive group could have altered the statistically significant association of less invasive serotypes with increasing age and comorbidities.

The final reasons why data on $S$ pneumoniae serotypes causing adult lung infection are important are the implications for prevention by vaccination. Although most of the serious $S$ pneumoniae infections occurring in adults are CAP, the adult $S$ pneumoniae vaccine (PPV, based on pneumococcal capsular polysaccharide from the commonest 23 serotypes) only prevents $S$ pneumoniae septicaemia and meningitis and has little efficacy against lung infection. ${ }^{11}$ The childhood PCV vaccine also uses capsular antigen but 
linked to a protein carrier so it is much more immunogenic in children than PPV. PCV has been highly successful in children, preventing invasive $S$ pneumoniae infections and colonisation with vaccine serotypes, and reducing the incidence of pneumonia. ${ }^{12}$ Due to herd immunity, PCV vaccination of children has also been associated with reduced incidence of $S$ pneumoniae CAP with vaccine serotypes in adults. $^{12}$ PCV coverage was originally limited to seven serotypes, but has recently increased to 13 . The increased immunogenicity of PCV over PPV (at least in children) and the evidence that it induces mucosal immunity suggests that vaccination of adults with PCV might prevent $S$ pneumoniae CAP. However, there are no data to support the use of PCV in adults as yet; large-scale trials are in progress. PPV and PCV only give serotypespecific protection, so identifying which serotypes cause CAP is clearly critical for assessing what the impact of PCV13 could be on adult CAP, and perhaps for choosing serotypes to be included in a future version of PCV for adults. Bewick et al show that if PCV is effective at preventing adult CAP, then using the PCV13 could prevent up to $57 \%$ of cases of disease. ${ }^{3}$ This proportion is probably an underestimate as in their study one-third of cases could not be serotyped and the multiplex assay is unlikely to be $100 \%$ sensitive. Hence even if the PCV13 vaccine only prevents a proportion of adult CAP caused by vaccine serotypes this still could have considerable effects on respiratory morbidity and mortality. As a consequence, the results of the current trials of PCV in adults are of great importance. A theoretical bonus of PCV vaccination in adults could be prevention of infective exacerbations of COPD. However, PCV13 serotypes cause only $43 \%$ of infective exacerbations of COPD associated with $S$ pneumoniae, ${ }^{10}$ and whether PCV can improve mucosal immunity within a damaged bronchus adequately to prevent infective exacerbations needs to be established.

$S$ pneumoniae is a nasopharyngeal commensal, and infants are thought to be the major reservoir for infection due to their high levels of colonisation. Routine childhood vaccination in the UK since
2006 with PCV has resulted in dramatic falls in the prevalence of vaccine serotypes as commensals, including serotype $14^{13}$ Hence the discovery by Bewick et al that serotype 14 was still the commonest cause of adult CAP is surprising, ${ }^{3}$ and perhaps suggests that the relatively low level of $S$ pneumoniae colonisation of adults maybe an alternative reservoir for infection. Colonisation of adults with vaccine serotypes could therefore lead to re-emergence of these serotypes in children if they are no longer included in a future PCV vaccine. Another important consideration is the incredibly rapid expansion of nonvaccine serotypes since the introduction of PCV, which has limited the impact of PCV on the overall incidence of $S$ pneumoniae infection. ${ }^{13}$ PCV13 includes many of the serotypes that have increased in incidence since introduction of the PCV7, so may help counter these changes in $S$ pneumoniae ecology. However, a lower proportion of adult CAP is caused by PCV13 serotypes than paediatric or invasive disease. This larger reservoir of non-vaccine serotypes makes serotype-replacement disease in response to adult vaccination with PCV more likely to be a major problem than it has been in children. Any introduction of PCV into adult populations will need to be supported by a post-vaccination surveillance program.

The data presented by Bewick et al on capsular serotypes causing adult CAP is a significant breakthrough. The sensitivity of the multiplex immunoassay makes this technology a potentially useful diagnostic test for $S$ pneumoniae CAP, and knowing the infecting serotype could help clinicians identify patients at higher risk of important complications. If trials demonstrate a beneficial effect against lung infection of PCV in adults, identifying serotypes causing CAP will be an essential tool for choosing which serotypes should be included in future PCV preparations and for monitoring changes in $S$ pneumoniae ecology in response to vaccination.

Contributors Written entirely by JSB.

Funding JSB works at UCLH/UCL which receives a proportion of funding from the Department of Health's NIHR Biomedical Research Centres funding scheme. His research programme is supported by the MRC, the Rosetrees Trust and a UCL Impact PhD fellowship.
Correction notice This article has been corrected since it was published online first. The name Berwick has been updated to Bewick.

Competing interests JSB received a travel grant from GSK to attend the American Thoracic Society conference in 2011.

Provenance and peer review Commissioned; internally peer reviewed.

Published Online First 12 March 2012

Thorax 2012;67:473-474

doi:10.1136/thoraxjnl-2012-201739

\section{REFERENCES}

1. Brown JS. Geography and the aetiology of community acquired pneumonia. Respirology 2009:14:1068-71.

2. Hyams C, Camberlein E, Cohen JM, et al. The Streptococcus pneumoniae capsule inhibits complement activity neutrophil phagocytosis by multiple mechanisms. Infect Immun 2010;78:704-15.

3. Bewick T, Sheppard C, Greenwood S, et al. Serotype prevalence in adults hospitalised with pneumococcal non-invasive community-acquired pneumonia. Thorax 2012;67:540-5.

4. Hyams C, Yuste J, Bax K, et al. Streptococcus pneumoniae resistance to complement-mediated immunity is dependent on capsular serotype. Infect Immun 2010;78:716-25.

5. Weinberger DM, Trzciński K, Lu YJ, et al. Pneumococcal capsular polysaccharide structure predicts serotype prevalence. PLoS Pathog 2009;5: e1000476.

6. Sanchez CJ, Hinojosa CA, Shivshankar P, et al Changes in capsular serotype alter the surface exposure of pneumococcal adhesins and impact virulence. PLoS One 2011;6:e26587.

7. Sleeman KL, Griffiths D, Shackley F, et al. Capsula serotype-specific attack rates and duration of carriage of Streptococcus pneumoniae in a population children. $\mathrm{J}$ Infect Dis 2006;194:682-8.

8. Weinberger DM, Harboe ZB, Sanders EA, et al. Association of serotype with risk of death due to pneumococcal pneumonia: a meta-analysis. Clin Infect Dis 2010;51:692-9.

9. Strachan $\mathbf{R E}$, Cornelius A, Gilbert GL, et al. Bacteria causes of empyema in children, Australia, 2007-2009. Emerg Infect Dis 2011;17:1839-45.

10. Domenech A, Ardanuy C, Calatayud L, et al. Serotypes and genotypes of Streptococcus pneumoniae causing pneumonia and acute exacerbations in patients with chronic obstructive pulmonary disease. J Antimicrob Chemother 2011;66:487-93.

11. Moberley SA, Holden J, Tatham DP, et al. Vaccines for preventing pneumococcal infection in adults. Cochrane Database Syst Rev 2008;(1):CD000422.

12. Dinleyici EC, Yargic ZA. Pneumococcal conjugated vaccines: impact of PCV-7 and new achievements in the postvaccine era. Expert Rev Vaccines 2008; 7:1367-94

13. Miller E, Andrews NJ, Waight PA, et al. Herd immunity and serotype replacement 4 years after seven-valent pneumococcal conjugate vaccination in England and Wales: an observational cohort study. Lancet Infect Dis 2011;11:760-8. 